\title{
The Effect of Non-Homogenous Perforation Pattern on Sound Absorption Bandwidth of Micro-Perforate Panel
}

\author{
Iwan Prasetiyo \\ Engineering Physics, Institut Teknologi Bandung, Jl.Ganesa No.10 \\ Bandung, 40132, Indonesia \\ Joko Sarwono \\ Engineering Physics, Faculty of Industrial Technology, Institut Teknologi Bandung, \\ Jl.Ganesa No.10 Bandung, 40132, Indonesia \\ E-mail: jsarwono@tf.itb.ac.id \\ Indra Sihar \\ Engineering Physics, Faculty of Industrial Technology, Institut Teknologi Bandung, \\ Jl.Ganesa No.10 Bandung, 40132, Indonesia \\ E-mail: sihar.indra@gmail.com
}

\begin{abstract}
Absorption bandwidth of conventional Micro-Perforated Panel backed by air cavity is limited around 1 octave bands particularly for pore diameter close to $1 \mathrm{~mm}$. Compared with porous material, the absorption bandwidth is relatively narrow. Hence, its applications may become limited. This research shows that multiple-peaks as result of the use of the non-homogenous perforation pattern are useful in extending the absorption bandwidth. An analytical model is used to predict the absorption coefficient while experimental results are provided for validation.
\end{abstract}

Keywords: Micro-perforated panel, non-homogenous perforation pattern, extending absorption bandwidth, excess sound absorber

\section{Introduction}

The applications of Micro-perforated panel (MPP) as sound absorber in various areas have been widely found such as room acoustics ${ }^{1,2}$, environmental noise abatement $^{3}$ and duct noise control ${ }^{4}$, since the formulation proposed by Dah You Maa in $1975 .{ }^{5}$ MPPs are usually placed in front of rigid wall with a particular air cavity in which the Helmholtz resonance mechanism is taken account for. Moreover, simple construction is another advantage of the MPP from point of practical concerns. Considering these facts, MPPs can be an alternative to traditional porous materials. However, unlike the porous materials, the performance of the MPP is usually limited to around one to two octaves. This narrow absorption bandwidth is as consequence of the Helmholtz resonance mechanism where its absorption is dependent on the perforation parameters while the maximum absorption occurs at the resonant frequency $f_{0}$.

Regarding with the effort of widening the absorption bandwidth, some studies can be found from literatures. Theoretically, the basic principles related with this objective were given by Dah You Ma. ${ }^{6}$ From his study, the absorption bandwidth become wider as the value acoustic reactance $\omega_{0} m$ is smaller while the value of the acoustic resistance $r$ is larger. This approach leads to 3 or 4 octave half- absorption bandwidth.

Qian J, Y. et al. ${ }^{7}$ conducted experimental study by reducing the perforation diameter to less than 100 $\mu m$ in order to get larger value of acoustic resistance. It is found that half-absorption bandwidth of 3-4 octaves with the peak absorption higher than 0.85 are pronounced. The results is promising in terms of practical purposes but facing difficulty in manufacturing technique to fabricate such tiny holes. Hence, in practical point of view, it would take time until an 
appropriate technology is ready to deal with the requirement otherwise it would be costly.

Jung et al. ${ }^{8}$ was focused to develop double and triple layer systems connected in series to obtain multiple of absorption peaks in which a wider absorption bandwidth of more than 4 octaves can be found accordingly. A similar approach can also be found from study conducted by Sakagami et al. ${ }^{9}$ from which a detail analysis with Helmholtz-Kirchhoff is provided. This approach is outperformed compared with single layer but more space required implementing the construction which is no always applicable in practice, e,g, room interior surface in buildings. Moreover, in terms of optimization, it is difficult as the relation between the acoustic impedance of each sub-system is not clear.

The use of multiple MPPs connected in parallel was also proposed. This can introduce multiple peaks so that wider absorption bandwidth can be expected. Some results have been demonstrated by Sakagami. ${ }^{10}$ in virtue of periodic structure theory and considering excess sound absorption on boundary due to the discountinity of impedance. Meanwhile, Sum et al. ${ }^{11}$ use parallelcircuit rule by considering interaction/coupling between the reflected sound field exist have demonstrated parallel MPP in widening absorption bandwidth.

Alternatively, air cavity can also be filled with porous material to extend the absorption bandwidth ${ }^{12}$. Wang, C, et al. ${ }^{13}$ proposed irregular-shaped cavity rather than exploiting the perforation parameter in order to get wider absorption bandwidth. This approach allows more acoustic modes in cavity coupled with air motion in MPP compared with rectangular-shaped cavity. Hence, more resonant modes over frequency can be expected so that the absorption bandwidth becomes wider.

In this manuscript, the effect of the inhomogeneous perforation pattern using two different MPP is investigated. It is considered to exploit further the effect of the multiple absorption peaks in order to allow the absorption bandwidth to become wider while the pore diameter is close to $1 \mathrm{~mm}$ and can not be reduced further due to the limitation of manufacturing technology. This situation causes the absorption bandwidth usually only around 1 octave

\section{MPP Theoretical Model}

\subsection{Impedance model for perforates}

Following Crandall theoretical model for short tube. ${ }^{14}$, viscous effect of narrow tubes can be quantified by defining the specific impedance of a single circular tube that is ratio of the sound pressure difference between the end of tube $(\Delta p)$ and average particle velocity across the tube cross section $(\bar{u})$. This gives

$$
Z=\frac{\Delta p}{\bar{u}}=j \omega \rho_{0} t\left[1-\frac{2}{k \sqrt{-j}} \frac{J_{1}(k \sqrt{-j})}{J_{0}(k \sqrt{-j})}\right]^{-1}
$$

where $k=r_{0} \sqrt{\rho_{0} \omega / \eta}$ is the perforate constant of perforation with $r_{0}$ the radius of tube, $\rho_{0}$ the density of air, $\eta$ the coefficient of viscosity. $J_{1}$ is the Bessel function of the first kind and first order and $J_{0}$ is the same of zeroeth order. For the case of short tubes of finite length (i.e. perforated plates) an end correction should be taken account.

\subsection{Impedance model for micro-perforated panels}

As the perforation diameter is reduced to sub-milimeter, the formulation in section 2.1 becomes the microperforated problem. Ma. ${ }^{5}$ first proposed an approximate model to calculate sound absorption of the microperforated panel. The model considers the microperforated panel consisting of parallel connected tubes in which the impedance of a single perforated hole as defined by Eq. (1). For normal incidence, the wave motion in all the short tubes can be regarded to be in phase and additive. Therefore, the relative acoustic impedance with considering the end correction based on Morse Ingard's model. ${ }^{15}$, this yields. ${ }^{6}$

$$
z=r+j x_{m}
$$

where

$$
\begin{aligned}
& r=\frac{32 \eta t}{\sigma \rho_{0} c d^{2}}\left(\left[1+\frac{k^{2}}{32}\right]^{1 / 2}+\frac{\sqrt{2}}{32} k \frac{d}{t}\right) \\
& x_{m}=\frac{\omega t}{\sigma c}\left(1+\left[1+\frac{k^{2}}{2}\right]^{-1 / 2}+0.85 \frac{d}{t}\right)
\end{aligned}
$$


For this formulation, perforation constant $k$ is defined as

$$
k=d \sqrt{\omega \rho_{0} / 4 \eta}
$$

where $d$ is the pore diameter, $\omega$ is the angular frequency, $\rho_{0}$ is the air density and $\eta$ is the coefficient of viscosity. The perforation ratio is given by

$$
\sigma=\frac{\pi}{4}\left(\frac{d}{b}\right)^{2}
$$

where $b$ is the separation of separations. It should be noted that the formulation above is valid for $19.6 \%$ perforation otherwise the effect of interaction between hole should be taken account. Moreover, the panel thickness is made to comparable to pore diameter in which the bending stiffness of panel becomes meaningless. This leads to the neglecting of flexibility of panel to be accepted.

MPP absorbers require a backing cavity with the depth of cavity $D$ as shown in Fig. 1. The air in the cavity acts as "air-cushion" to the vibration of air in the tiny holes of the MPP. Therefore, the vibration energy is damped and a relatively broadband sound absorption can be obtained accordingly. The combined surface impedance of the MPP and the air in the cavity $z_{m p p}$ is thus expressed as

$$
z_{m p p}=r+j\left(x_{m}-\cot \frac{\omega D}{c}\right)
$$

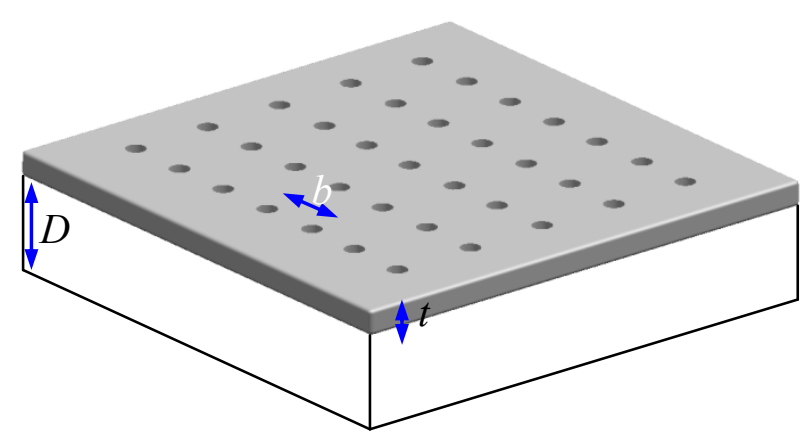

Fig. 1. Illustration of the MPP backed by air cavity

For normal incidence, the sound absorption coefficient is defined as

$$
\alpha=\frac{4 r}{(1+r)^{2}+\left(x_{m}-\cot \omega D / c\right)^{2}}
$$

\subsection{Impedance model for non-homogenous perforation pattern}

To enhance the absorption bandwidth, a nonhomogenous perforation pattern is considered. This MPP consists of sub-MPP with different perforation parameters particularly the perforation ratio.

As discontinuity of surface impedance is present for the non-homogenous perforation pattern, the wave diffraction phenomena exists accordingly. This introduces excess sound absorption on the boundary surface. Takahasi. ${ }^{16}$ treated this problem as a scattering wave of the boundary surface on which the discontinuity of impedance exists.

Raleigh model. ${ }^{17}$ can be used to find the solution of the scattered field. The incident plane wave $\psi_{i}$ with unit amplitude expressed in terms of the velocity potential and time factor $e^{-j \omega t}$ omitted throughout is

$$
\psi_{i}(x, z)=e^{j\left(\alpha_{0} x-\gamma_{0} z\right)}
$$

where $\alpha_{0}=\kappa \sin \theta$ and $\gamma_{0}=\kappa \cos \theta \quad$ with $\kappa=2 \pi / \lambda$ and $\theta$ incident angle. A scattered wave $\psi_{s}$ is expressed with unknown coefficient $\psi_{m}$ as

$$
\psi_{s}(x, y)=\sum_{m=-\infty}^{\infty} \psi_{m} e^{j\left(\alpha_{m} x+\gamma_{m} z\right)}
$$

where $\alpha_{m}=\alpha_{0}+2 m \pi / L$ and $\gamma_{m}=\sqrt{k^{2}-\alpha_{m}^{2}}$ with $L$ the period of surface.

The boundary condition represented by the specific acoustic admittance $A$ of each surface at $z=0$ is

$$
\frac{\partial}{\partial z}\left[\psi_{i}+\psi_{s}\right]+j A k\left[\psi_{i}+\psi_{s}\right]=0, \text { on } z=0
$$

where

$$
A=\left\{\begin{array}{c}
A_{1}, \quad 0 \leq x<l \\
A_{2}, \quad l \leq x<L
\end{array}\right.
$$

For this case, the respective $A_{1}$ and $A_{2}$ correspond to acoustic admittance of each MPP generally expressed as $A=z^{-1}$ with $z$ as defined in Eq. (2). Substituting Eq. (9) and (10) into Eq. (11) gives

$$
\sum_{m=-\infty}^{\infty} \boldsymbol{\Psi}_{m}\left(\gamma_{m}+A k\right) e^{j 2 m \pi x / L}=\gamma_{0}-A k
$$


Applying the one-dimensional Fourier expansion and rearranging the result using an infinite set of simulatenous equation with respect to $m$, this yields

$$
\sum_{m} \Psi_{m} C_{m n}=D_{n}
$$

where

$$
\begin{gathered}
C_{m n}=\frac{1}{L} \int_{0}^{L}\left(\gamma_{m}+A k\right) e^{-j 2(n-m) \pi x / L} d x \\
D_{n}=\frac{1}{L} \int_{0}^{L}\left(\gamma_{0}-A k\right) e^{-j 2 n \pi x / L} d x
\end{gathered}
$$

The absorption coefficient of boundary surface is regarded as the energy fraction flowing outward in normal direction to surface. Considering this concept, the angle dependent is

$$
\alpha_{\theta}=1-\sum_{\operatorname{Re}\left[\gamma_{m}\right]}\left|\boldsymbol{\Psi}_{m}\right|^{2} \frac{\gamma_{m}}{\gamma_{0}}
$$

\section{Experimental Method}

The measurement of absorption coefficient of MPP was conducted using impedance tube according to ISO 10534-2. ${ }^{18}$ (see Fig. 2). The white noise was generated in sound source and the travelling plane waves through a10 $\mathrm{cm}$ radius tube were picked up using two microphones. From this, the transfer impedance can be determined and the sound absorption coefficient for frequency ranging from $64 \mathrm{~Hz}-1.6 \mathrm{KHz}$ can be obtained accordingly.

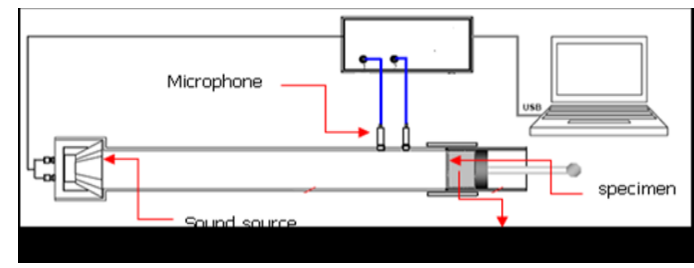

Fig. 2. Experimental setup used for measuring absorption coefficient of MPP

Detail of specimens can be observed from Fig. 3 while the parameters of each MPP are listed in Table 1 . It should be noted the pore diameter is chosen $0.9 \mathrm{~mm}$ as this is the smallest pore diameter can be formed by the manufacturing technology found by authors.

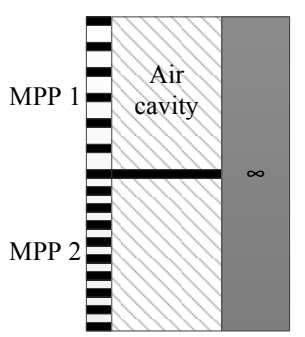

Fig. 3. Sketch of specimen

Table 1. MPP parameters of specimens

\begin{tabular}{cccccc}
\hline & Material & $t(\mathrm{~mm})$ & $\sigma(\%)$ & $d(\mathrm{~mm})$ & $D(\mathrm{~mm})$ \\
\hline MPP1 & acrylic & 1.5 & 0.79 & 0.9 & 17 \\
MPP2 & acrylic & 1.5 & 2.07 & 0.9 & 17
\end{tabular}

\section{Results and Discussion}

Fig. 4 presents normal absorption of non-homogenous perforation pattern. It is clear that two absorption peaks are pronounced from analytical results at $753 \mathrm{~Hz}$ and $1189 \mathrm{~Hz}$ as indicated by solid curve. The presence of those peaks is expected as two perforation ratios are considered in the MPP. Hence, two Helmholtz resonance mechanisms work when incoming wave imping on the MPP surface.

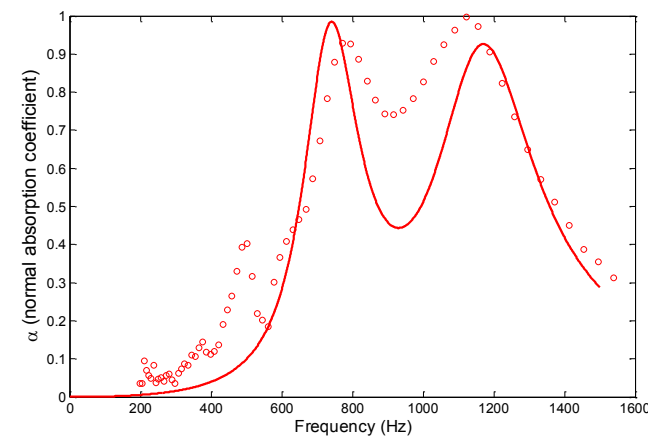

Fig. 4. Normal absorption coefficient of non-homogenous perforation pattern as combination of $0.79 \%$ and $2.07 \%$ perforation ratio.

The presence of the two peaks is also present in the measurement result as marked by circle as shown in Fig. 4. However, another peak is also seen from the measurement around $502 \mathrm{~Hz}$ besides the peaks at 753 $\mathrm{Hz}$ and $1189 \mathrm{~Hz}$. This peak corresponds with fundamental mode of the panel rather than caused by the Helmholtz resonance. This is so as the panel 
vibration can not be totally omitted from specimen while such vibration is neglected from analytical model by assuming the panel is rigid instead on elastic one. Bravo et al. ${ }^{19}$, addressed such phenomena in MPP.

The large discrepancy in absorption is clearly seen at frequency of $850 \mathrm{~Hz}$ to $1050 \mathrm{~Hz}$ where the measured absorption coefficient around 0.74 which is higher than that of the analytical result which is only found 0.44 as the lowest one. The results are getting closer in agreement when the period of surface $L$ reduces as indicated in Fig. 5. This approach is opted to allow representing the actual condition of measurement where the periodic surface of specimen is quite small in 100 $\mathrm{cm}$ disc. This result indicates that the effect of the excess sound absorption is more significant as $L$ get smaller than the sound wavelength.

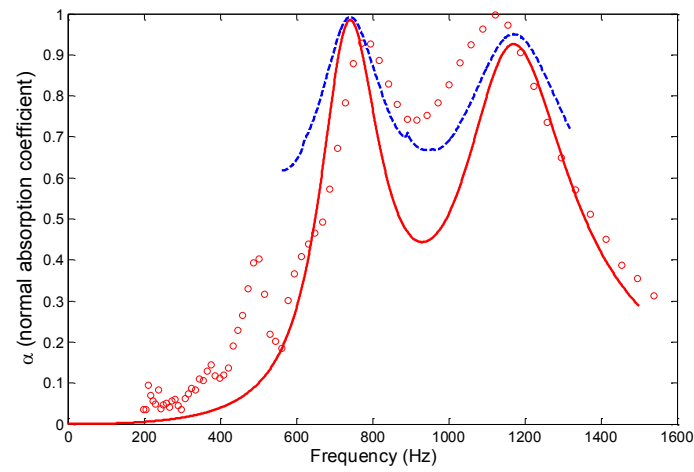

Fig.5 The effect of reducing the surface periodic on the absorption coefficient.

Compared with conventional MPP from which single perforation ratio employed, the use of non-homogenous perforation pattern has a wider absorption bandwidth as shown in Fig. 6. It is found that the $0.79 \%$ perforation ratio provides the half-absorption bandwidth of $373 \mathrm{~Hz}$ while that of $2.07 \%$ perforation ratio is $511 \mathrm{~Hz}$. Meanwhile, the non-homogenous pattern as combination of $0.79 \%$ and $2.07 \%$ perforation ratio can provide the half-absorption bandwidth of $710 \mathrm{~Hz}$ which is higher than 1 octave.

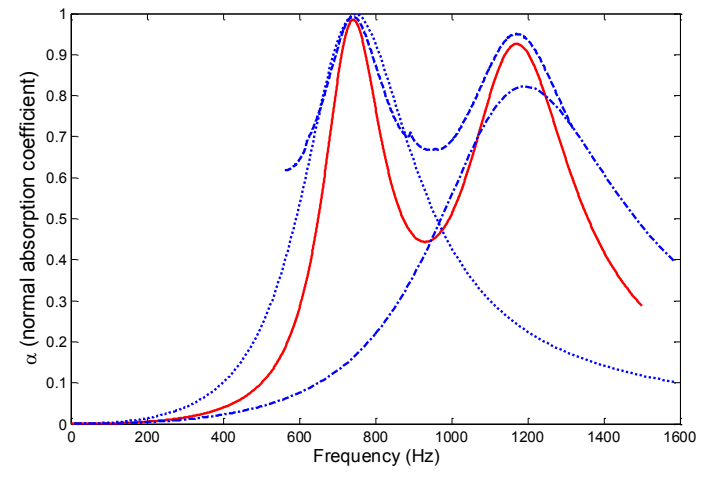

Fig. 6. Absorption bandwidth comparison between homogenous perforation and non-homogenous perforation

\section{Conclusion}

Compared with conventional MPP from which single perforation ratio employed, the use of non-homogenous perforation pattern is useful in extending the absorption bandwidth. Care need to be taken as the effect of excess sound absorption is more significant when the ratio of periodic surface to sound wavelength get smaller. Moreover, as panel vibration cannot be totally omitted particularly for non-rigid panel so that the absorption peak obtained are related to either Panel controlled or Helmholtz controlled resonances.

\section{Acknowledgements}

This research is funded by Institut Teknologi Bandung through Innovation research and research group program.

\section{References}

1. Fuchs HV, Zha X. Acrylic-glass sound absorbers in plenum of the Deutscher Bundestag. ApplAcoust 1997;51:211-7

2. Fuchs HV, Zha X. Micro-perforated structures as sound absorbers - a review and outlook. ActaAcoust 2006;92:139-46

3. Asdrubali F, Pispola G. Properties of transparent sound absorbing panels for innoise barrier. J AcoustSoc Am 2007;121:214-21.

4. Wu MQ. Micro-perforated panels for duct silencing. Noise Control Eng 1997;45:69-77

5. Maa DY. Theory and design of microperforated panel sound-absorbingconstructions. SciSinica 1975;17:55-71.

6. Maa DY. Potential of micro-perforated panel absorber. J AcoustSoc Am 1998;104:2861-6. 
7. Qian, Y, et al. Investigation on micro-perforated panel absorber with ultra-micro perforations, Applied Acoustics , 2013, 74, 931 - 935

8. Jung, S S, Kim, YT and Lee, D H, Sound Absorption of Micro-Perforated Panel, Journal of the Korean Physical Society, 2007, 50, 1044-1051

9. Sakagami, K.; Matsutani, K. \& Morimoto, M. Sound absorption of a double-leaf micro-perforated panel with an air-back cavity and a rigid-back wall: Detailed analysis with a Helmholtz-Kirchhoff integral formulation Applied Acoustics , 2010, 71, 411 - 417

10. Sakagami, K, et al. Pilot studi on wideband sound absorber obtained by combination of two different micrperforated panel (MPP) absorbers, Acoust. Sci. \& Tech. 30, 2 (2009)

11. Sum, K.S, et al, Use of parallel Microperforated Panel Subabsorber for Noise Control in Ducts, ICSV13, Vienna, (2006).

12. N. Atalla and F. Sgard, "Modeling of perforated plates and screens using rigid frame porous models," J. Sound Vib.303, 195-208 (2007)

13. Wang, C, Cheng, L, Yu, G, Sound absorption of a microperforated panel backed by an irregular-shaped cavity, J AcoustSoc Am 2010;127:238

14. Crandall IB. Theory of vibrating systems and sound. New York: D. Van Nostrand\& Co. Inc.; 1927. pp. 229-241.

15. P. M. Morse and U. Ingard, Theoretical Acoustics, McGraw-Hill, New York, 1968, pp. 460-463.

16. D. Takahashi, "Excess sound absorption due to periodically arranged absorptive materials,'”J. Acoust. Soc. Am., 66, p. 2215-2222 (1989).

17. Lord Rayleigh, "On the dynamical theory of gratings," Proc. R. Soc. London A 79, 399-416 (1907)

18. ISO 10534-2, Acoustics - Determination of sound absorption coefficient and impedance in impedance tubes - Part 2: Transfer-function method

19. Teresa Bravo, Cédric Maury and CédricPinhède,Vibroacoustic properties of thin micro-perforated panel absorbers J. Acoust. Soc. Am. 132, 789 (2012) 